\title{
Exact Radial Natural Frequencies of Functionally Graded Hollow Long Cylinders
}

\author{
Vebil Yıldirım \\ Department of Mechanical Engineering, University of Cukurova, 01330, Adana, Turkey \\ E-mail address: vebil@cu.edu.tr \\ ORCID numbers of authors: \\ 0000-0001-9955-8423 \\ Received date: August 2017 \\ Accepted date: December 2017
}

\begin{abstract}
In this work the exact free axisymmetric pure radial vibration of hollow infinite cylinders made of hypothetically functionally power-graded materials having identical inhomogeneity indexes for both Young's modulus and the material density is addressed. The equation of motion is obtained as a linear second-order Bessel's ordinary differential equation with constant coefficients based on the axisymmetric linear elasticity theory. For traction free boundaries, a closed form frequency equation is offered. After verifying the present results for cylinders made of both isotropic and homogeneous materials, and isotropic functionally graded materials, an extensive parametric study is carried out to investigate the influences of both the thickness and inhomogeneity indexes on the natural frequencies. Results are presented in both graphical and tabular forms. It was revealed that the fundamental frequency in the radial mode is principally affected from the inhomogeneity parameters than the higher ones. However, the natural frequencies except the fundamental ones are dramatically affected from the thickness of the cylinder. As the thickness decreases, the natural frequencies considerably increase. It is also revealed that, there is a linear relationship between the fundamental frequency and others in higher modes.
\end{abstract}

Keywords: Free vibration, natural frequency, thick-walled hollow cylinder, functionally graded.

\section{Introduction}

Vibration of thin/thick-walled cylinders is of great significance in many engineering applications such as pressure vessel, heat exchangers, nuclear reactor containments, various pipes and tubes. Pioneering studies concerning the vibration of cylinders date back late of $1800 \mathrm{~s}$. One of the earliest works on the vibration of cylinders was carried out by Chree [1]. Using the linear three dimensional elasticity theory, Greenspon [2] studied the flexural vibrations of infinitely long traction free hollow thick-walled cylinders. Gazis [3] studied the vibration of infinitely long traction free hollow cylinders on the basis of three dimensional elasticity theory. Gladwell and Tahbildar [4] solved the problem of axisymmetric vibrations of cylinders with the help of the finite-element method. Gladwell and Vijay [5] also analyzed the vibration of free finite length circular cylinders based on the finite element approach. Hutchinson [6] first handled the vibrations of finite length rods and solid cylinders on the basis of linear 3D elasticity and offered a semi-analytical highly accurate method to solve the problem. Then, Hutchinson and El-Azhari [7] applied the same method for the vibrations of free hollow finite length circular cylinders. By employing the energy method based on the 3D theory of elasticity, Singal and Williams [8] studied theoretically and experimentally the vibrations of thick hollow cylinders and rings. The axisymmetric stress-free vibration of a thick elastic cylinder has been studied under plane strain conditions by Gosh [9]. Gosh [9] obtained the solution for forced vibration by using the Laplace transform and presented natural frequency and dynamic stresses for various types of loading, Poisson's 
ratio and aspect ratios of the cylinder. Leissa and So [10], and So and Leissa [11] studied threedimensional analysis of the vibrations of free and cantilevered solid cylinders using simple algebraic polynomials in the Ritz method. Liew et al. [12] investigated the free vibrations of stress free hollow cylinders of arbitrary cross-section based on the three dimensional energy displacement expressions. Hung et al. [13] considered the free vibration of cantilevered cylinders. Wang and Williams [14] presented vibrational modes of thick-walled cylinders of finite length based on the finite element method. On the basis of linear 3D theory of elasticity, and by using the Ritz method and Chebyshev polynomials, Zhou et al. [15] worked on the vibration analysis of solid and hollow circular cylinders including rods and curved panels. In this general semi-analytical series solution having high accuracy and good convergence, offered by Zhou et al. [15], the technique of variables separation is developed for various boundary conditions. Mofakhami et al. [16] studied the free vibration of cylinders with finite length under fixed-fixed and free-free boundaries based on the solutions of infinite cylinders and the technique of separation of variables. Abbas [17] treated with the free vibration of a poroelastic hollow cylinder. Yahya and Abd-Alla [18] considered pure radial vibrations in an isotropic elastic hollow cylinder with rotation.

As time progresses and engineers familiarize themselves with new advanced materials such as anisotropic, functionally graded, carbon nanotube composites, studies have focused on the vibration problems of cylinders made of such advanced materials. From those Nelson et al. [19] worked on vibration and waves in laminated orthotropic circular cylinders. Vibration of anisotropic composite cylinders is addresses by Huang and Dong [20]. Yuan and Hsih [21] investigated three dimensional wave propagation in composite cylindrical shells. By using the Ritz method, Kharouf and Heyliger [22] presented a numerical method for finding approximate solutions to static and axisymmetric vibration problems for piezoelectric cylinders, including those composed of more than one material. Markus and Mead [23-24] studied both axisymmetric and asymmetric wave motion in orthotropic cylinders. Ding et al. [25-26] studied elasto-dynamic and thermoelastic-dynamic problems of a nonhomogeneous orthotropic hollow cylinders.

As to the functionally graded materials (FGM), Heyliger and Jilani [27] studied the free vibrations of inhomogeneous elastic cylinders and spheres. By using strains-displacement relations from Love's shell theory and the eigenvalue governing equation from Rayleigh-Ritz method, Loy et al. [28] presented a study on the vibration of cylindrical shells made of a functionally graded material (FGM) composed of stainless steel and nickel. The properties are graded in the thickness direction according to a volume fraction power-law distribution in Loy et al's [28] study. Their results showed that the frequency characteristics are similar to that observed for homogeneous isotropic cylindrical shells and the frequencies are affected by the constituent volume fractions and the configurations of the constituent materials. Han et al. [29] presented an analytical-numerical method for analyzing characteristics of waves in a cylinder composed of functionally graded material (FGM) by dividing the FGM cylinder into a number of annular elements with three-nodal-lines in the wall thickness. Han et al. [29] assumed a linear variation of material properties along the thickness direction and used the Hamilton principle to develop the dispersion equations for the cylinder. Their numerical results demonstrated that the ratio of radius to thickness has a stronger influence on the frequency spectra in the circumferential wave than on that in the axial wave. Patel et al. [30] studied the free vibration analysis of functionally graded elliptical cylindrical shells based on the higher-order theory. Pelletier and Vel [31] studied analytically the steady-state thermoelastic response of functionally graded orthotropic cylindrical shells. Arciniega and Reddy [32] considered a large deformation analysis of functionally graded shells. Yang and Shen [33] investigated the free vibration of shear deformable functionally graded cylindrical panels. Jianqiao et al. [34] dealt with wave propagation in nonhomogeneous magneto-electro-elastic hollow cylinders. Abd-Alla et al. [35] studied influences of the inhomogeneity on the composite infinite cylinder of isotropic material. Based on the first-order shear 
deformation theory and linear elasticity, Tornabene et al. [36] studied the dynamic behavior of functionally graded moderately thick conical, cylindrical shells and annular plates via the generalized differential quadrature (GDQ) method. They considered two different power-law distributions for the ceramic volume fraction. Keleş and Tutuncu [37] analytically performed free and forced vibration analyses of power-law graded hollow cylinders and spheres. Although their subject matters are out of the present study, it may be useful to cite some studies concerning the vibration of cylinders and cylindrical shells which are functionally graded with state-of-the-art technological structural materials [38-40] in the open literature.

As seen from the above literature survey, vibration problems of solid/hollow cylinders made of functionally graded materials are not studied widely over the time. This was the motivation to the author. As a fundamental work, the present study may be thought of as an extension of Gosh's [9] study to traction free cylinders made of isotropic functionally graded materials. In the present study, to achieve an analytical solution for natural frequencies in purely radial modes, the inhomogeneity indexes of both elasticity modulus and Poisson's ratio are assumed to be identical by necessity. As stated in the related section, otherwise, it is not possible to get a closed form solution. In these cases, the employment of any numerical procedure becomes compulsory. One of the aims of the present study is to have a rough idea about the general response of such cylinders to the free vibration before numerically treatment of the problem. In the parametric study, almost all the variables which substantially influence on the natural frequencies are considered except the effect of Poisson's ratio. In Gosh's study [9] the effect of Poisson's ratios on the natural frequencies was clearly disclosed. The numerical results given here may also be served as a benchmark solution to some advanced numerical studies.

\section{Clarification of the Problem}

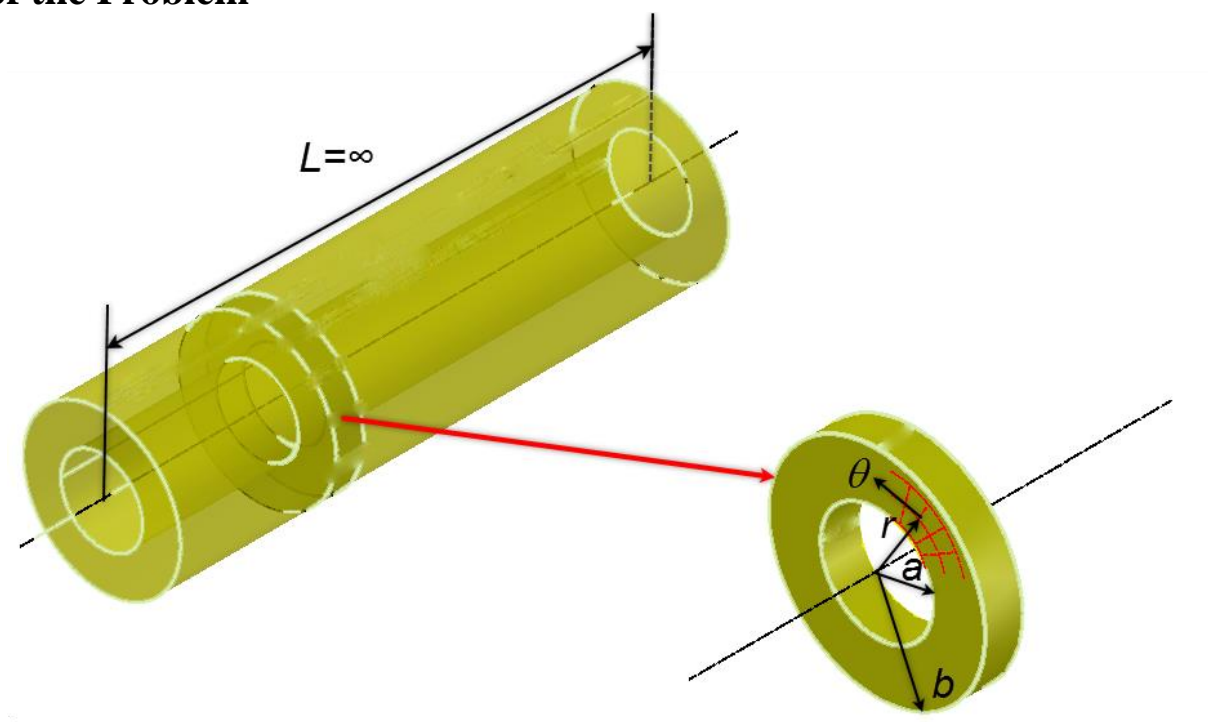

Fig. 1. Geometry of a cylinder

Let's consider a thick-walled hollow cylinder with inner radius $a$ and outer radius $b$ (Fig. 1). Under axisymmetric conditions, relationships between strain and displacement are written in polar coordinates as

$$
\varepsilon_{r}=\frac{d u_{r}}{d r} \quad ; \quad \varepsilon_{\theta}=\frac{u_{r}}{r}
$$


where the radial unit strain and the circumferential unit strain are symbolized by $\varepsilon_{r}$, and $\varepsilon_{\theta}$, respectively. In Eq. (1), the radial displacement is represented by $u_{r}$. If $\sigma_{r}$, and $\sigma_{\theta}$ signify the radial stress and the hoop stress, respectively, then Hooke's law for an infinite cylinder made of an isotropic and homogeneous linear elastic material is given by

$$
\begin{aligned}
& \sigma_{r}=C_{11}(r) \varepsilon_{r}+C_{12}(r) \varepsilon_{\theta} \\
& \sigma_{\theta}=C_{12}(r) \varepsilon_{r}+C_{11}(r) \varepsilon_{\theta}
\end{aligned}
$$

Where under plain strain assumptions

$$
\begin{aligned}
& C_{11}(r)=E(r) \frac{(1-v)}{(1+v)(1-2 v)} \\
& C_{12}(r)=E(r) \frac{v}{(1+v)(1-2 v)}
\end{aligned}
$$

For an isotropic but non-homogeneous FGM material, the material grading rule in the radial direction is assumed to obey the following simple power rule

$$
\begin{gathered}
E(r)=E_{b}\left(\frac{r}{b}\right)^{\eta} \\
\rho(r)=\rho_{b}\left(\frac{r}{b}\right)^{\eta}
\end{gathered}
$$

where $\rho$ is the material density. The material inhomogeneity index is denoted by $\eta ; E_{b}$ and $\rho_{b}$ are the reference values of the mixture of the material at the outer surface. Poisson's ratios of both graded materials are assumed to be unaffected along the radial direction as in the most of the related realm.

In Eq. (4) those properties do not completely correspond to a physical material since both Young's modulus and density are assumed to have the same inhomogeneity index. To get an analytical solution to the problem, as seen later, taking both inhomogeneity indexes as if they are identical is going to be inevitable because it is not possible to find a closed-form solution in other choices which require exactly numerical solution techniques.

If the body forces are neglected, the equation of motion in the radial direction is written as follows

$$
\frac{1}{r} \frac{\partial}{\partial r}\left(r \sigma_{r}\right)-\frac{\sigma_{\theta}}{r}=\rho \frac{\partial^{2} u_{r}}{\partial t^{2}}
$$

where $t$ is the time. Substitution both Eq. (1) and (2) together with Eq. (4) into Eq. (5) gives

$$
\frac{\partial^{2} u_{r}}{\partial r^{2}}+\left(\frac{1+\eta}{r}\right) \frac{\partial u_{r}}{\partial r}-\frac{\left(1-\eta \frac{C_{12}(r)}{C_{11}(r)}\right)}{r^{2}} u_{r}=-\left(\frac{\rho(r)}{C_{11}(r)}\right) \frac{\partial^{2} u_{r}}{\partial t^{2}}
$$

By assuming a harmonic motion, $u_{r}(r, t)=u_{r}^{*}(r) e^{i \omega t}$, with an angular velocity $\omega(\mathrm{rad} / \mathrm{s})$, Eq. (6) gives way to Bessel's differential equations [41-43]. 
where

$$
-\left(\frac{1-\eta\left(\frac{v}{1-v}\right)}{r^{2}}+\Omega^{2}\right) u_{r}^{*}+\left(\frac{1+\eta}{r}\right) \frac{d u_{r}^{*}}{d r}+\frac{d^{2} u_{r}^{*}}{d r^{2}}=0
$$

$$
\Omega=\sqrt{\frac{\rho(r)}{C_{11}(r)}} \omega=\sqrt{\frac{(1-2 v)(1+v) \rho_{b}}{(1-v) E_{b}}} \omega=\sqrt{\frac{\left(1-v-2 v^{2}\right) \rho_{b}}{(1-v) E_{b}}} \omega=\frac{\beta}{a}
$$

and $\beta$ is the dimensionless natural frequency. The solution to this equation, Eq. (7), is going to be in the form of [41-43]

$$
u_{r}^{*}(\mathrm{r})=r^{-\eta / 2}\left(C_{1} J_{\frac{\xi}{2}}(r \Omega)+C_{2} Y_{\frac{\xi}{2}}(r \Omega)\right)
$$

where $C_{1}$ and $C_{2}$ are arbitrary constants and $J_{\frac{\xi}{2}}(r \Omega)$ and $Y_{\frac{\xi}{2}}(r \Omega)$ denote Bessel's functions of the first and second kind of order $\frac{\xi}{2}$, respectively, and $\xi$ is

$$
\xi=\sqrt{4+\eta^{2}-4 \eta\left(\frac{v}{1-v}\right)}=\sqrt{\frac{\left(-4-\eta^{2}+(2+\eta)^{2} v\right)}{(v-1)}}
$$

The first derivative of the solution of the radial displacement, $u_{r}^{*}$, and the radial stress, $\sigma_{r}^{*}$, may be obtained in terms of integration constants, $C_{1}$ and $C_{2}$, as follows

$$
\begin{gathered}
\frac{d u_{r}^{*}}{d r}=r^{-\eta / 2}\left(\frac{1}{2} C_{1} \Omega\left(J_{\frac{\xi}{2}-1}(r \Omega)-J_{\frac{\xi}{2}+1}(r \Omega)\right)+C_{2} \Omega\left(Y_{\frac{\xi}{2}-1}(r \Omega)-Y_{\frac{\xi}{2}+1}(r \Omega)\right)\right) \\
-\frac{1}{2} \eta r^{-\frac{\eta}{2}-1}\left(C_{1} J_{\frac{\xi}{2}}(r \Omega)+C_{2} Y_{\frac{\xi}{2}}(r \Omega)\right) \\
\sigma_{r}^{*}(r)=\frac{E_{b} r^{-\frac{\eta}{2}-1}\left(\frac{r}{b}\right)^{\eta}}{2\left(2 v^{2}+v-1\right)}\left(C_{1}(\eta-(\eta+2) v) J_{\frac{\xi}{2}}(r \Omega)+C_{1}(v-1) r \Omega J_{\frac{\xi-2}{2}}(r \Omega)-C_{1}(v-1) r \Omega J_{\frac{\xi+2}{2}}(r \Omega)\right. \\
\left.+C_{2}(\eta-(\eta+2) v) Y_{\frac{\xi}{2}}(r \Omega)+C_{2}(v-1) r \Omega Y_{\frac{\xi-2}{2}}(r \Omega)-C_{2}(v-1) r \Omega Y_{\frac{\xi+2}{2}}(r \Omega)\right)
\end{gathered}
$$

Eqs. (9) and (11) are used when applying the boundary conditions given at both surfaces. The radial displacement vanishes, $u_{r}^{*}=0$, at the fixed surface while the radial stress becomes zero, $\sigma_{r}^{*}=0$, at the free surface. For instance, if surface-free boundaries are considered then one may obtain

$$
\left\{\begin{array}{l}
\sigma_{r}^{*}(a) \\
\sigma_{r}^{*}(b)
\end{array}\right\}=\left[\begin{array}{ll}
A_{1,1} & A_{1,2} \\
A_{2,1} & A_{2,2}
\end{array}\right]\left\{\begin{array}{l}
C_{1} \\
C_{2}
\end{array}\right\}=\boldsymbol{A}\left\{\begin{array}{l}
C_{1} \\
C_{2}
\end{array}\right\}=\left\{\begin{array}{l}
0 \\
0
\end{array}\right\}
$$

To get non-trivial solutions, natural frequencies are determined from the frequencies which make the determinant of the characteristic coefficient matrix, $\boldsymbol{A}$, zero. Considering traction-free boundary conditions for a hollow infinite cylinder made of a hypothetically functionally power-graded material, elements of the coefficient matrices are found in closed forms as follows 


$$
\begin{aligned}
A_{1,1}= & \frac{a^{-\frac{\eta}{2}-1}\left(\frac{a}{b}\right)^{\eta}\left(a(v-1) \Omega J_{\frac{\xi-2}{2}}(a \Omega)+(\eta-(\eta+2) v) J_{\frac{\xi}{2}}(a \Omega)-a(v-1) \Omega J_{\frac{\xi+2}{2}}(a \Omega)\right) E_{b}}{2\left(2 v^{2}+v-1\right)} \\
A_{1,2}= & \frac{a^{-\frac{\eta}{2}-1}\left(\frac{a}{b}\right)^{\eta}\left(a(v-1) \Omega Y_{\frac{\xi-2}{2}}(a \Omega)+(\eta-(\eta+2) v) Y_{\frac{\xi}{2}}(a \Omega)-a(v-1) \Omega Y_{\frac{\xi+2}{2}}(a \Omega)\right) E_{b}}{2\left(2 v^{2}+v-1\right)} \\
A_{2,1}= & \frac{b^{-\frac{\eta}{2}-1}\left(b(v-1) \Omega J_{\frac{\xi-2}{2}}(b \Omega)+(\eta-(\eta+2) v) J_{\frac{\xi}{2}}(b \Omega)-b(v-1) \Omega J_{\frac{\xi+2}{2}}(b \Omega)\right) E_{b}}{2\left(2 v^{2}+v-1\right)} \\
A_{2,2}= & \frac{\frac{\eta}{2}-1\left(b(v-1) \Omega Y_{\frac{\xi-2}{2}}(b \Omega)+(\eta-(\eta+2) v) Y_{\frac{\xi}{2}}(b \Omega)-b(v-1) \Omega Y_{\frac{\xi+2}{2}}(b \Omega)\right) E_{b}}{2\left(2 v^{2}+v-1\right)}
\end{aligned}
$$

\section{Authentication of the Formulation}

To confirm the present dimensionless frequencies, as a first example, a hollow infinite thin-walled cylinder made of an isotropic and homogeneous material is considered. The first ten natural frequencies are listed in Table 1. As seen from this table there is full agreement with those of Gosh's [9] frequencies.

As a second example a hollow infinite cylinder made of a hypothetically power-law graded material is taken into account. Results, which are all based on the similar procedure, are tabulated in Table 2 in a comparative manner. In this table, $\eta=0$ corresponds a cylinder made of an isotropic and homogeneous material. It is seen from Table 2 that present results are in good harmony with the others. Figure 2 shows also the determinant-dimensionless frequency curve for $\eta=-5$, and $b / a=2$.

Table 1. Comparison of the present non-dimensional results with the open literature for thin-walled cylinder made of an isotropic and homogeneous material $\left(v=0.3 ; \frac{b}{a}=1.02\right)$

\begin{tabular}{lll}
\hline & Present & \multicolumn{1}{c}{$[9]$} \\
\hline$\beta_{1}$ & 0.894602 & 0.89 \\
$\beta_{2}$ & 157.082 & 157.10 \\
$\beta_{3}$ & 314.161 & 314.20 \\
$\beta_{4}$ & 471.24 & 471.20 \\
$\beta_{5}$ & 628.319 & 628.30 \\
$\beta_{6}$ & 785.399 & 785.40 \\
$\beta_{7}$ & 942.478 & 942.50 \\
$\beta_{8}$ & 1099.56 & 1100.00 \\
$\beta_{9}$ & 1256.64 & 1257.00 \\
$\beta_{10}$ & 1413.72 & 1414.00 \\
\hline
\end{tabular}


Table 2. Comparison of the present FGM results with the open literature $(b / a=2, v=0.3)$.

\begin{tabular}{|c|c|c|c|c|c|c|c|c|c|c|c|}
\hline & \multicolumn{2}{|c|}{$\eta=-5$} & \multicolumn{2}{|c|}{$\eta=-2$} & \multicolumn{3}{|c|}{$\eta=0$} & \multicolumn{2}{|c|}{$\eta=2$} & \multicolumn{2}{|c|}{$\eta=5$} \\
\hline & Present & [37] & Present & [37] & resent & [9] & [37] & Present & [37] & Present & [37] \\
\hline$\beta_{1}$ & 0.752017 & 0.77435 & 0.684418 & 0.69407 & 0.633263 & 0.6335 & 0.63563 & 0.586987 & 0.58309 & 0.537493 & 0.52547 \\
\hline$\beta_{2}$ & 3.4389 & 3.43985 & 3.20038 & 3.20181 & 3.21655 & 3.218 & 3.21793 & 3.3714 & 3.37244 & 3.81843 & 3.81886 \\
\hline$\beta_{3}$ & 6.44741 & 6.44802 & 6.31288 & 6.31356 & 6.3193 & 6.319 & 6.31999 & 6.40267 & 6.40331 & 6.66528 & 6.66578 \\
\hline$\beta_{4}$ & 9.53654 & 9.53698 & 9.44462 & 9.44508 & 9.44864 & 9.449 & 9.44910 & 9.50494 & 9.50538 & 9.6854 & 9.68579 \\
\hline$\beta_{5}$ & 12.6508 & 12.65115 & 12.5813 & 12.58161 & 12.5842 & 12.58 & 12.58455 & 12.6266 & 12.62695 & 12.7634 & 12.76368 \\
\hline$\beta_{6}$ & 15.7758 & 15.77602 & 15.7199 & 15.72016 & 15.7222 & 15.72 & 15.72249 & 15.7562 & 15.75647 & 15.8661 & 15.86638 \\
\hline$\beta_{7}$ & 18.9062 & 18.90638 & 18.8595 & 18.85972 & 18.8614 & 18.86 & 18.86165 & 18.8898 & 18.89000 & 18.9816 & 18.98183 \\
\hline$\beta_{8}$ & 22.0397 & 22.03991 & & 21.99987 & 22.0013 & 22.00 & 22.00151 & 22.0256 & 22.02583 & 22.1045 & 22.10466 \\
\hline$\beta_{9}$ & 25.1753 & 25.17544 & 25.1402 & 25.14037 & 25.1416 & 25.14 & 25.14180 & 25.1629 & 25.16309 & 25.232 & 25.23214 \\
\hline$\beta_{10}$ & 28.3122 & 28.31231 & 28.281 & 28.28112 & 28.2822 & 28.28 & 28.28239 & 28.3012 & 28.30131 & 28.3626 & 28.36273 \\
\hline
\end{tabular}

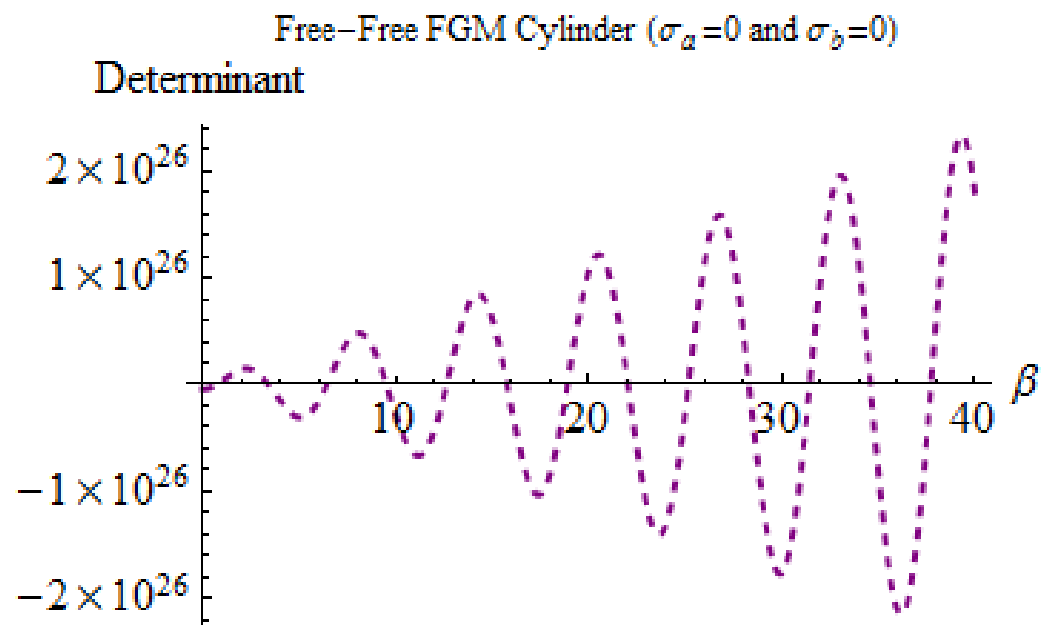

Fig. 2. Determinant-dimensionless frequency curve for $\eta=-5$, and $b / a=2$

\section{Effects of the Aspect Ratios and Material Gradients on the Natural Frequencies}

In this section a FGM cylinder of $v=0.3$ is considered. The functionally graded material (FGM) is taken to be a hypothetical one exhibiting significant inhomogeneity.

Variation of the natural frequencies with the aspect ratio, which is defined as the ratio of the outer and inner radii, and inhomogeneity indexes is presented in Tables 3 and 4. Table 3 indicates the influence of the aspect ratios and inhomogeneity indexes, which is defined in Eq. (4), on the first ten natural frequencies of a FGM free-free infinite cylinder for $\eta=-5, \eta=0, \eta=7$, and $\eta=10$. Table 4 shows the influence of the aspect ratios and inhomogeneity indexes on the first three natural frequencies of a FGM free-free infinite cylinder for some inhomogeneity indexes whose values are changed from $\eta=$ -4 to $\eta=5$ with an increase by the unit, except $\eta=0$. 
Table 3. The influence of the aspect ratios and inhomogeneity indexes on the first ten natural frequencies of a FGM free-free infinite cylinder $(v=0.3)$ for $\eta=-5, \eta=0, \eta=7$, and $\eta=10$

\begin{tabular}{|c|c|c|c|c|c|c|c|c|c|c|}
\hline$b / a$ & 1.02 & 1.03 & 1.04 & 1.05 & 1.075 & 1.10 & 1.25 & 1.50 & 1.75 & 2.00 \\
\hline & \multicolumn{10}{|c|}{$\eta=-5$} \\
\hline$\beta_{1}$ & 0.894748 & 0.890568 & 0.886514 & 0.882583 & 0.873263 & 0.864622 & 0.82422 & 0.785036 & 0.764088 & 0.752017 \\
\hline$\beta_{2}$ & 157.093 & 104.74 & 78.566 & 62.8643 & 41.9354 & 31.4778 & 12.7008 & 6.49988 & 4.45582 & 3.4389 \\
\hline$\beta_{3}$ & 314.166 & 209.449 & 157.093 & 125.68 & 83.7996 & 62.8628 & 25.2007 & 12.6787 & 8.52027 & 6.44741 \\
\hline$\beta_{4}$ & 471.243 & 314.166 & 235.628 & 188.506 & 125.68 & 94.2684 & 37.7445 & 18.9249 & 12.6628 & 9.53654 \\
\hline$\beta_{5}$ & 628.322 & 418.884 & 314.166 & 251.336 & 167.564 & 125.679 & 50.2996 & 25.1894 & 16.8278 & 12.6508 \\
\hline$\beta_{6}$ & 785.401 & 523.603 & 392.704 & 314.166 & 209.449 & 157.092 & 62.8591 & 31.4613 & 21.0022 & 15.7758 \\
\hline$\beta_{7}$ & 942.48 & 628.322 & 471.243 & 376.997 & 251.335 & 188.506 & 75.421 & 37.737 & 25.1813 & 18.9062 \\
\hline$\beta_{8}$ & 1099.56 & 733.041 & 549.782 & 439.828 & 293.222 & 219.92 & 87.9841 & 44.0147 & 29.3632 & 22.0397 \\
\hline$\beta_{9}$ & 1256.64 & 837.761 & 628.322 & 502.659 & 335.109 & 251.335 & 100.548 & 50.2939 & 33.5468 & 25.1753 \\
\hline \multirow[t]{2}{*}{$\beta_{10}$} & 1413.72 & 942.48 & 706.861 & 565.49 & 376.996 & 282.75 & 113.112 & 56.5739 & 37.7316 & 28.3122 \\
\hline & \multicolumn{10}{|c|}{$\eta=0$} \\
\hline$\beta_{1}$ & 0.894602 & 0.890244 & 0.885946 & 0.881708 & 0.871366 & 0.861367 & 0.807616 & 0.736002 & 0.67955 & 0.633263 \\
\hline$\beta_{2}$ & 157.082 & 104.724 & 78.5453 & 62.8386 & 41.8978 & 31.4289 & 12.595 & 6.33146 & 4.25189 & 3.21655 \\
\hline$\beta_{3}$ & 314.161 & 209.442 & 157.082 & 125.667 & 83.7808 & 62.8383 & 25.147 & 12.5902 & 8.40834 & 6.3193 \\
\hline$\beta_{4}$ & 471.24 & 314.161 & 235.621 & 188.498 & 125.667 & 94.2521 & 37.7086 & 18.8654 & 12.5868 & 9.44864 \\
\hline$\beta_{5}$ & 628.319 & 418.88 & 314.161 & 251.329 & 167.554 & 125.667 & 50.2726 & 25.1446 & 16.7704 & 12.5842 \\
\hline$\beta_{6}$ & 785.399 & 523.6 & 392.7 & 314.161 & 209.441 & 157.082 & 62.8375 & 31.4254 & 20.9562 & 15.7222 \\
\hline$\beta_{7}$ & 942.478 & 628.319 & 471.24 & 376.992 & 251.329 & 188.498 & 75.403 & 37.707 & 25.1429 & 18.8614 \\
\hline$\beta_{8}$ & 1099.56 & 733.039 & 549.779 & 439.824 & 293.217 & 219.913 & 87.9687 & 43.9891 & 29.3302 & 22.0013 \\
\hline$\beta_{9}$ & 1256.64 & 837.759 & 628.319 & 502.656 & 335.104 & 251.329 & 100.535 & 50.2714 & 33.5179 & 25.1416 \\
\hline \multirow[t]{2}{*}{$\beta_{10}$} & 1413.72 & 942.478 & 706.859 & 565.487 & 376.992 & 282.745 & 113.1 & 56.5539 & 37.7059 & 28.2822 \\
\hline & \multicolumn{10}{|c|}{$\eta=7$} \\
\hline$\beta_{1}$ & 0.894397 & 0.88979 & 0.885153 & 0.880487 & 0.868724 & 0.856851 & 0.785445 & 0.676595 & 0.587642 & 0.517028 \\
\hline$\beta_{2}$ & 157.133 & 104.799 & 78.6447 & 62.9616 & 42.0777 & 31.6628 & 13.0978 & 7.11859 & 5.18815 & 4.22088 \\
\hline$\beta_{3}$ & 314.186 & 209.479 & 157.132 & 125.729 & 83.8709 & 62.9557 & 25.4038 & 13.0114 & 8.93897 & 6.92433 \\
\hline$\beta_{4}$ & 471.257 & 314.186 & 235.654 & 188.539 & 125.727 & 94.3304 & 37.8805 & 19.1498 & 12.9492 & 9.8673 \\
\hline$\beta_{5}$ & 628.332 & 418.899 & 314.185 & 251.36 & 167.599 & 125.726 & 50.4017 & 25.3589 & 17.0445 & 12.9023 \\
\hline$\beta_{6}$ & 785.409 & 523.615 & 392.72 & 314.185 & 209.478 & 157.129 & 62.9409 & 31.5972 & 21.1763 & 15.9782 \\
\hline$\beta_{7}$ & 942.487 & 628.332 & 471.256 & 377.013 & 251.359 & 188.537 & 75.4891 & 37.8503 & 25.3268 & 19.0754 \\
\hline$\beta_{8}$ & 1099.57 & 733.05 & 549.794 & 439.842 & 293.243 & 219.947 & 88.0425 & 44.112 & 29.488 & 22.1851 \\
\hline$\beta_{9}$ & 1256.64 & 837.768 & 628.332 & 502.671 & 335.127 & 251.358 & 100.599 & 50.379 & 33.6561 & 25.3026 \\
\hline \multirow[t]{2}{*}{$\beta_{10}$} & 1413.72 & 942.487 & 706.87 & 565.501 & 377.012 & 282.771 & 113.158 & 56.6496 & 37.8288 & 28.4255 \\
\hline & \multicolumn{10}{|c|}{$\eta=10$} \\
\hline$\overline{\beta_{1}}$ & 0.89431 & 0.889597 & 0.884814 & 0.879968 & 0.86761 & 0.854972 & 0.777305 & 0.66029 & 0.568707 & 0.498278 \\
\hline$\beta_{2}$ & 157.178 & 104.866 & 78.733 & 63.0709 & 42.2372 & 31.8695 & 13.5291 & 7.74807 & 5.88568 & 4.9228 \\
\hline$\beta_{3}$ & 314.209 & 209.513 & 157.176 & 125.783 & 83.9511 & 63.06 & 25.6302 & 13.3752 & 9.38644 & 7.42197 \\
\hline$\beta_{4}$ & 471.272 & 314.208 & 235.684 & 188.575 & 125.781 & 94.4001 & 38.0328 & 19.3995 & 13.2639 & 10.2267 \\
\hline$\beta_{5}$ & 628.343 & 418.916 & 314.208 & 251.387 & 167.639 & 125.778 & 50.5163 & 25.5481 & 17.2851 & 13.1796 \\
\hline$\beta_{6}$ & 785.418 & 523.628 & 392.738 & 314.207 & 209.51 & 157.171 & 63.0327 & 31.7493 & 21.3705 & 16.203 \\
\hline$\beta_{7}$ & 942.494 & 628.343 & 471.271 & 377.031 & 251.386 & 188.572 & 75.5657 & 37.9774 & 25.4894 & 19.2641 \\
\hline$\beta_{8}$ & 1099.57 & 733.059 & 549.806 & 439.857 & 293.265 & 219.977 & 88.1082 & 44.2211 & 29.6278 & 22.3475 \\
\hline$\beta_{9}$ & 1256.65 & 837.776 & 628.343 & 502.685 & 335.147 & 251.385 & 100.657 & 50.4746 & 33.7787 & 25.4452 \\
\hline$\beta_{10}$ & 1413.73 & 942.494 & 706.88 & 565.513 & 377.03 & 282.794 & 113.209 & 56.7346 & 37.9379 & 28.5524 \\
\hline
\end{tabular}


Table 4. The influence of the aspect ratios and inhomogeneity indexes on the first three natural frequencies of a FGM free-free infinite cylinder $(v=0.3)$ for $\eta=-4,-3,-2,-1,1,2,3,4$, and 5

\begin{tabular}{|c|c|c|c|c|c|c|c|c|c|c|}
\hline$b / a$ & 1.02 & 1.03 & 1.04 & 1.05 & 1.075 & 1.10 & 1.25 & 1.50 & 1.75 & 2.00 \\
\hline & \multicolumn{10}{|c|}{$\eta=-4$} \\
\hline$\beta_{1}$ & 0.894719 & 0.890503 & 0.8864 & 0.882408 & 0.872884 & 0.863973 & 0.820964 & 0.775819 & 0.748964 & 0.731791 \\
\hline$\beta_{2}$ & 157.088 & 104.732 & 78.5558 & 62.8516 & 41.9168 & 31.4536 & 12.6484 & 6.41608 & 4.35369 & 3.32657 \\
\hline$\beta_{3}$ & 314.163 & 209.446 & 157.088 & 125.674 & 83.7903 & 62.8507 & 25.1741 & 12.6348 & 8.46472 & 6.38368 \\
\hline & \multicolumn{10}{|c|}{$\eta=-3$} \\
\hline$\beta_{1}$ & 0.89469 & 0.890438 & 0.886287 & 0.882233 & 0.872505 & 0.863323 & .81766 & 0.766172 & 0.732559 & 0.709067 \\
\hline$\beta_{2}$ & 157.084 & 104.726 & 78.5486 & 62.8427 & 41.9037 & 31.4366 & 12.6114 & 6.35679 & 4.28103 & 3.24623 \\
\hline$\beta_{3}$ & 314.161 & 209.443 & 157.084 & 125.669 & 83.7837 & 62.8422 & 25.1554 & 12.604 & 8.42558 & 6.33871 \\
\hline & \multicolumn{10}{|c|}{$\eta=-2$} \\
\hline$\beta_{1}$ & 0.89466 & 0.890373 & 0.886173 & 0.882058 & 0.872125 & 0.862671 & & 0.756219 & 0.715205 & 0.684418 \\
\hline$\beta_{2}$ & 157.082 & 104.723 & 78.5444 & 62.8375 & 41.8962 & 31.4 & 12.5 & 6.32 & 4.23937 & 3.20038 \\
\hline$\beta_{3}$ & 314.16 & 209.441 & 157.082 & 125.667 & 83.78 & 62.8373 & 25.1447 & 12.5862 & 8.40307 & 6.31288 \\
\hline & \multicolumn{10}{|c|}{$\eta=-1$} \\
\hline$\beta_{1}$ & 0.894631 & 0.890308 & 0.88606 & 0.881883 & 0.871746 & 0.862019 & & & 0.697358 & 0.658769 \\
\hline$\beta_{2}$ & 881 & 104. & 78.5 & & 943 & & & & 4.22961 & 3.19044 \\
\hline$\beta_{3}$ & .16 & 209.441 & 157.081 & 125.666 & 83.779 & 62.836 & 25.1419 & 12.5816 & 8.39732 & 6.30639 \\
\hline & \multicolumn{10}{|c|}{$\eta=1$} \\
\hline$\beta_{1}$ & 0.894573 & 0.890179 & 0.885833 & 0.881534 & 0.870986 & 0.860715 & 279 & 0.726062 & 2312 & \\
\hline$\beta_{2}$ & & & & & & & & & 563 & \\
\hline$\beta_{3}$ & 314.162 & 209.443 & 157.085 & 125.67 & 83.7853 & 62.8443 & 25.16 & 12.6119 & 8.43609 & 6.35149 \\
\hline & \multicolumn{10}{|c|}{$\eta=2$} \\
\hline$\beta_{1}$ & 0.894543 & 0.890114 & 719 & 0.88 & 0.870607 & 0.860065 & & & 107 & 0.58698 \\
\hline$\beta_{2}$ & 7.089 & 104.734 & 78.5584 & & 41.9216 & & & & 4.38954 & 3.3714 \\
\hline$\beta_{3}$ & 314.164 & 209.447 & 157.089 & 125.675 & 83.7927 & 62.8538 & 25.181 & 466 & 8.48039 & 6.40267 \\
\hline & \multicolumn{10}{|c|}{$\eta=3$} \\
\hline$\beta_{1}$ & 0.894514 & 0.890049 & 0.885606 & & 0.870228 & 0.859416 & & & 274 & \\
\hline$\beta_{2}$ & 157.095 & 104.742 & 78.5695 & 62.8686 & 41.9418 & 31.4861 & & & 4.50181 & 3.49505 \\
\hline$\beta_{3}$ & 314.167 & 209.451 & 157.094 & 125.682 & 83.8028 & 62.867 & 25.2099 & 12.6943 & 8.54101 & 6.47243 \\
\hline & \multicolumn{10}{|c|}{$\eta=4$} \\
\hline$\beta_{1}$ & 0.894485 & 0.889984 & 0.885492 & & 0.86985 & 0.85877 & 0.794525 & 0.698641 & 0.618006 & 0.5512 \\
\hline$\beta_{2}$ & .102 & 104.753 & 78.5837 & & 41.9675 & 31.5196 & 12.792 & 6.64832 & 4.64025 & 3.64521 \\
\hline$\beta_{3}$ & 4.17 & 209.456 & 157.102 & 125.691 & 83.8156 & 62.8838 & 25.2466 & 12.7548 & 8.61761 & 6.56019 \\
\hline & \multicolumn{10}{|c|}{$\eta=5$} \\
\hline$\beta_{1}$ & 0.894456 & 0.88992 & 0.885379 & 0.880835 & 0.869474 & 0.858127 & 0.791408 & 0.690634 & 0.606359 & 0.537493 \\
\hline$\beta_{2}$ & 157.111 & 104.766 & 78.601 & 62.9076 & 41.9987 & 31.5602 & 12.8794 & 6.78502 & 4.80243 & 3.81843 \\
\hline$\beta_{3}$ & 314.175 & 209.463 & 157.11 & 125.702 & 83.8313 & 62.9041 & 25.2912 & 12.828 & 8.70979 & 6.66528 \\
\hline
\end{tabular}



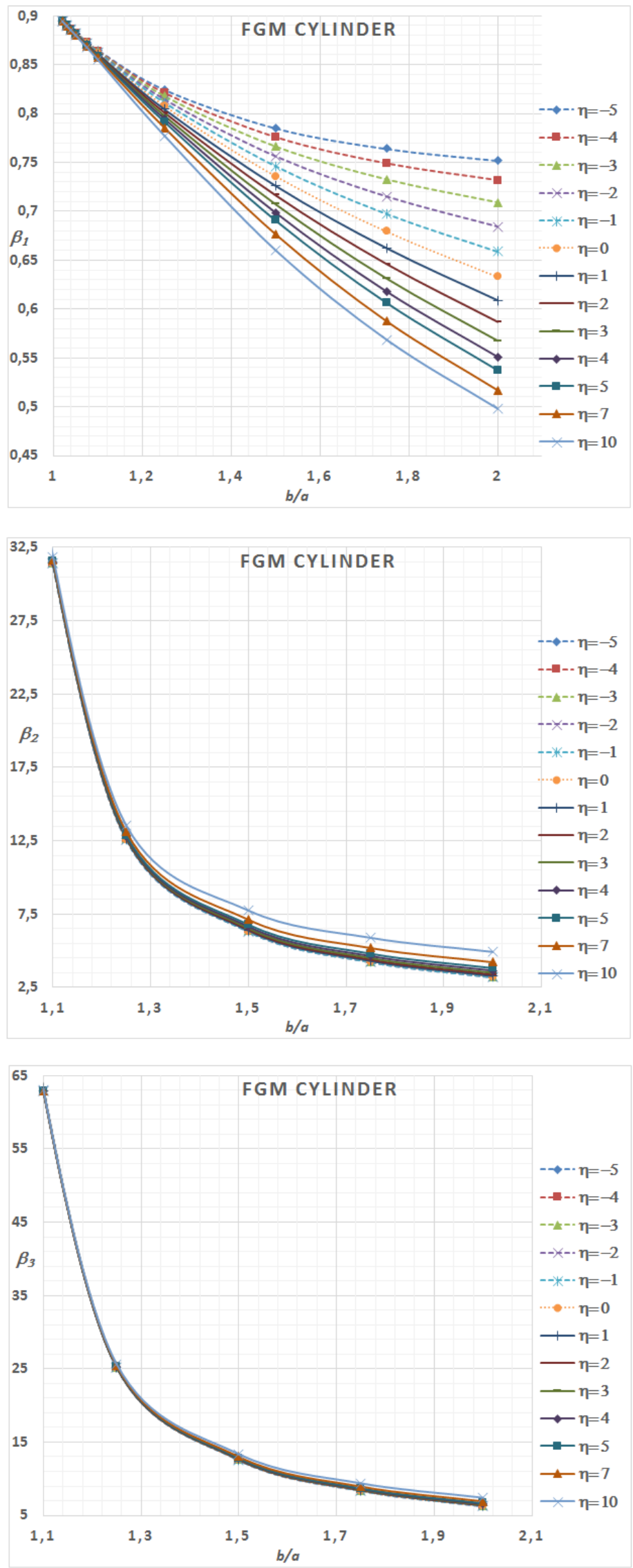

Fig. 3. Variation of the first three natural frequencies with the inhomogeneity indexes for $1.1 \leq b / a \leq 2$ and $-5 \leq \eta \leq 10$. 

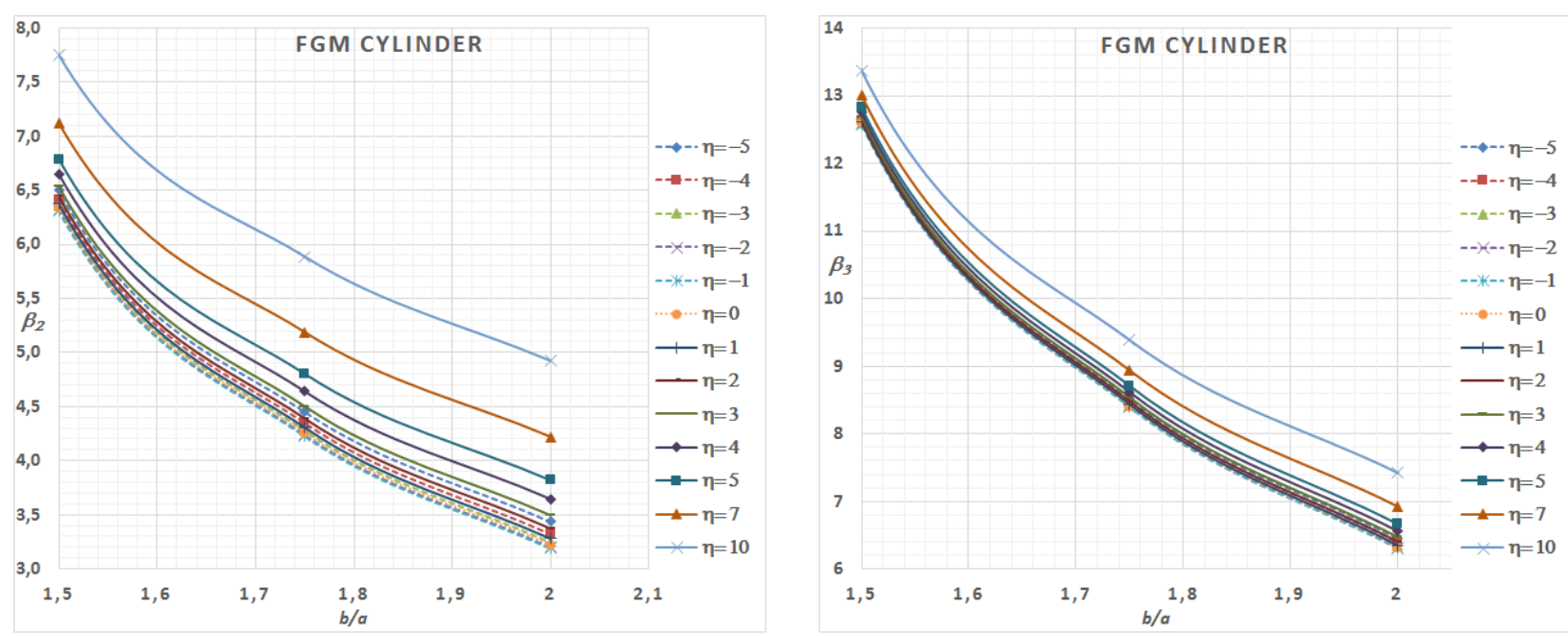

Fig. 4. Variation of the second and the third natural frequencies with the inhomogeneity indexes for $1.5 \leq b / a \leq 2$ and $-5 \leq \eta \leq 10$.

Both Table 3 and Table 4 suggest that as the thickness increases, the dimensionless natural frequencies decrease. This response is slightly observed for fundamental frequencies. However, there is sharply decrease in frequencies of higher modes as the thickness build up.

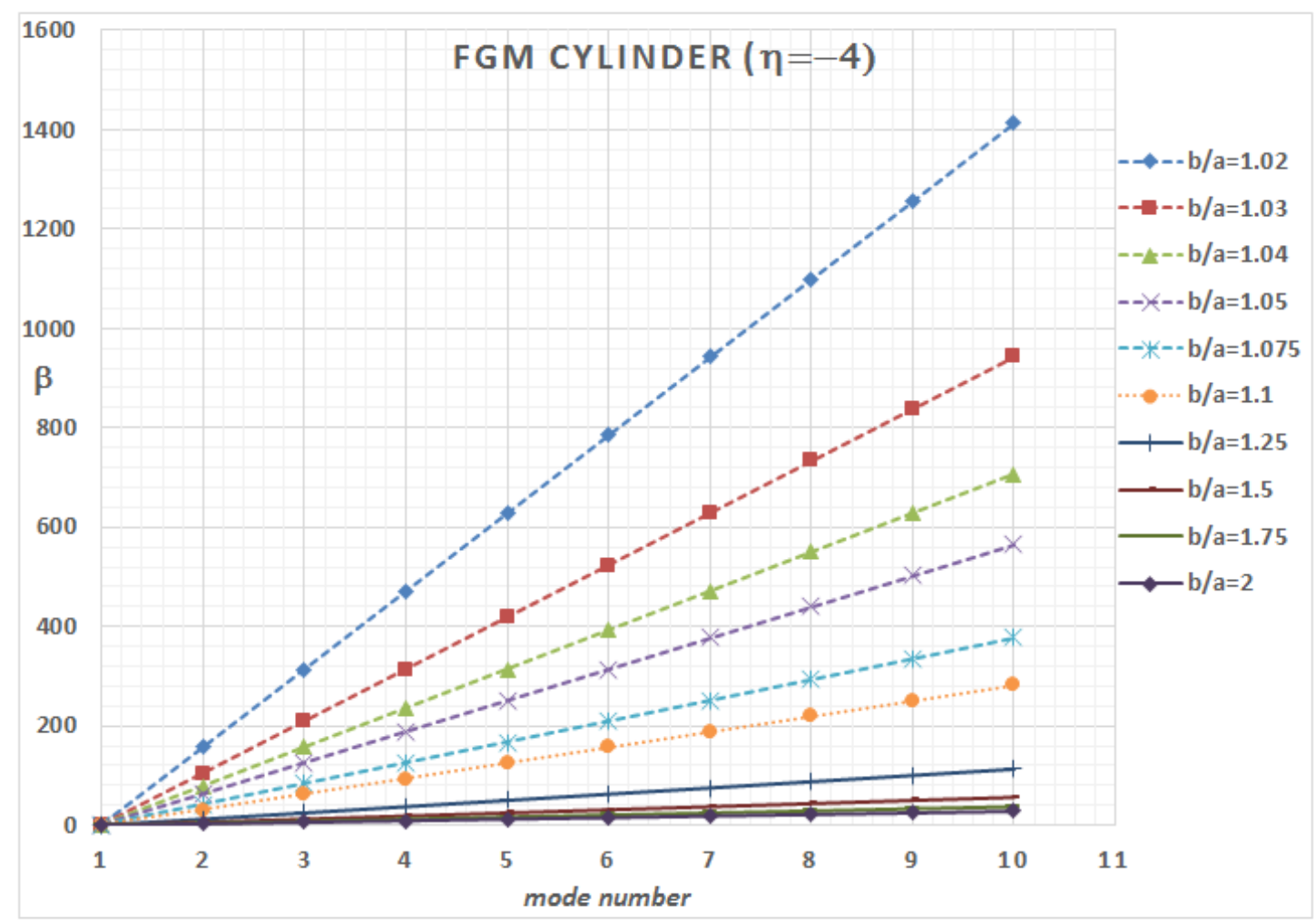

Fig. 5. The relationship between the frequencies of the same cylinder 
For the dimensionless frequencies, the degree of to be influenced from the change of inhomogeneity indexes is rather slow. This is demonstrated in Figs. 3 and 4. Figure 3 shows the variation of the first three natural frequencies with the inhomogeneity indexes for $1.1 \leq b / a \leq 2$ and $-5 \leq \eta \leq 10$. Variation of the second and the third natural frequencies with the inhomogeneity indexes for $1.5 \leq$ $b / a \leq 2$ and $-5 \leq \eta \leq 10$ is illustrated in Fig. 4. As seen from Fig. 3, the fundamental frequencies are more affected from the change in the inhomogeneity index. In the interval of the aspect ratio, $1.1 \leq$ $b / a \leq 1.5$, it is almost impossible to observe the variation of natural frequencies in higher modes with the change in the inhomogeneity index. However, as in Fig. 4, this may be clearly observed for $1.5 \leq$ $b / a \leq 2$ for the second and third frequencies.

In this study, it is also revealed that, there is a linear relationship between the pure radial fundamental frequency and others in higher modes of the same cylinder for all inhomogeneity indexes and aspect ratios as seen from Fig. 5.

\section{Concluding Remarks}

In the present study, the free vibration of hypothetically power-law graded hollow infinite stress-free cylinders is analytically studied to investigate the influences of inhomogeneity indexes and aspect ratios, whose figures are chosen in the broadest possible range of values that can be used in practice, on the natural frequencies. The following conclusions are drawn:

- The fundamental frequency is mainly affected from the variation of the inhomogeneity index than those of other higher frequencies. This may be acceptable as plausible because of huge differences between the fundamental and higher frequencies. For example, for $b / a=1.02$ the natural frequency in the second mode is more than 150 times the fundamental frequency, however it is around four times the first one for $b / a=2$ (see Tables 3-4).

- The effects on the frequencies of the changes in thickness of the cylinder are clearly observed. Increasing thickness produce a substantial decrease in dimensionless frequencies which are particularly in frequencies in higher modes.

- There is also a linear relationship between the frequencies of fundamental and higher modes of the same cylinder.

As stated before, the present results pertaining to the cylinders made of hypothetically FGM materials are obtained by using the same inhomogeneity index for both elasticity modulus and material density. It is a great possibility that there is no such a physical material that provides this feature. For this sense, it may be useful to confirm the present conclusions with physical materials. As mentioned above, this requires the putting numerical solution techniques into practice.

\section{References}

[1] Chree, C., The equations of an isotropic elastic solid in polar and cylindrical coordinates, their solutions and applications. Transactions of the Cambridge Philosophical Society, 14, 250-309, 1889.

[2] Greenspon, J. E., Flexural vibrations of a thick-walled circular cylinder according to the exact theory of elasticity. Journal of Aero/Space Sciences, 27, 1365-1373, 1957.

[3] Gazis, D. C., Three dimensional investigation of the propagation of waves in hollow circular cylinder. Journal of the Acoustical Society of America, 31, 568-578, 1959. 
[4] Gladwell, G. M., Tahbildar, U. C., Finite element analysis of axisymmetric vibrations of cylinders. Journal of Sound and Vibration, 22, 143-157, 1972.

[5] Gladwell, G. M., Vijay, D. K., Natural frequencies of free finite length circular cylinders. Journal of Sound and Vibration, 42, 387-397, 1975.

[6] Hutchinson, J. R., Vibrations of solid cylinders. Journal of Applied Mechanics, 47, 901-907, 1980.

[7] Hutchinson, J. R., El-azhari, S. A., Vibrations of free hollow circular cylinders. Journal of Applied Mechanics, 53, 641-646, 1986.

[8] Singal, R. K., Williams, K. A., Theoretical and experimental study of vibrations of thick circular cylindrical shell and rings. Journal of Vibration, Acoustics, Stress and Reliability in Design, 110, 533-537, 1988.

[9] Gosh, A. K., Axisymmetric vibration of a long cylinder. Journal of Sound and Vibration, 186(5), 711-721, 1995.

[10] Leissa, A. W., So, J., Accurate vibration frequencies of circular cylinders from threedimensional analysis. Journal of the Acoustical Society of America, 98, 2136-2141, 1995.

[11] So, J., Leissa, A. W., Free vibration of thick hollow circular cylinders from three-dimensional analysis. Journal of Vibration and Acoustics, 119, 89-95, 1997.

[12] Liew, K. M., Hung, K. C., Lim, M. K., Vibration of stress free hollow cylinders of arbitrary cross-section. Journal of Applied Mechanics, ASME, 62, 718-724, 1995.

[13] Hung, K. C., Liew, K. M., Lim, M. K., Free vibration of cantilevered cylinders: effects of cross-sections and cavities. Acta Mechanica, 113, 37-52, 1995.

[14] Wang, H., Williams, K. Vibrational modes of thick cylinders of finite length. Journal of Sound and Vibration, 191, 955-971, 1996.

[15] Zhou, D., Cheung, Y. K., Lo, S. H., Au, F. T. K., 3D vibration analysis of solid and hollow circular cylinders via Chebyshev-Ritz method. Computer Methods in Applied Mechanics and Engineering, 192, 1575-1589, 2003.

[16] Mofakhami, M. R., Toudeshky, H. H., Hashemi, S. H. H., Finite cylinder vibrations with different end boundary conditions. Journal of Sound and Vibration, 297, 293-314, 2006.

[17] Abbas, İ., Natural frequencies of a poroelastic hollow cylinder. Acta Mechanica, 186(1-4), 229-237, 2006.

[18] Yahya, G. A., Abd-Alla, A. M., Radial vibrations in an isotropic elastic hollow cylinder with rotation. Journal of Vibration and Control, 22(13), 3123-3131, 2016.

[19] Nelson, R. B., Dong, S. B., Kalra, R. D., Vibrations and waves in laminated orthotropic circular cylinders. Journal of Sound and Vibration, 18, 429-444, 1971.

[20] Huang, K. H., Dong, S. B., Propagating waves and edge vibrations in anisotropic composite cylinders. Journal of Sound and Vibration, 96, 363-379, 1984.

[21] Yuan, F. G., Hsieh, C. C., Three-dimensional wave propagation in composite cylindrical shell. Composite Structures, 42, 153-167, 1988.

[22] Kharouf, N., Heyliger, P. R., Axisymmetric free vibrations of homogeneous and laminated piezoelectric cylinders. Journal of Sound and Vibration, 174(4), 539-561, 1994.

[23] Markus, S., Mead, D. J., Axisymmetric and asymmetric wave motion in orthotropic cylinders. Journal Sound Vibration, 181, 127-147, 1995.

[24] Markus, S., Mead, D. J., Wave motion in a 3-layered, orthotropic isotropic orthotropic composite shell. Journal Sound Vibration, 181, 149-167, 1995.

[25] Ding, H. J., Wang, H. M., Chen, W. Q., Elastodynamic solution of a non-homogeneous orthotropic hollow cylinder. Acta Mechanica Sinica, 18, 621-628, 2002.

[26] Ding, H. J., Wang, H. M., Chen, W. Q., A solution of a non-homogeneous orthotropic cylindrical shell for axisymmetric plane strain dynamic thermo elastic problems. Journal of Sound and Vibration, 263, 815-829, 2003. 
[27] Heyliger, P. R., Jilani, A., The free vibrations of inhomogeneous elastic cylinders and spheres. International Journal of Solids and Structures, 29(22), 2689-2708, 1992.

[28] Loy, C. T., Lam, J. N., Reddy, J. N., Vibration of functionally graded cylindrical shells. Int. J. Mech. Sci. 41, 309-324, 1999.

[29] Han, X., Liu, G. R., Xi, Z. C., Lam, K. Y., Characteristics of waves in a functionally graded cylinder. Int. Journal for Numerical Methods in Engineering, 53(3), 653-676, 2002.

[30] Patel, B. P., Gupta, S. S., Loknath, M. S., Kadu, C. P., Free vibration analysis of functionally graded elliptical cylindrical shells using higher-order theory. Compos. Struct., 69, 259-270, 2005.

[31] Pelletier, J. L., Vel, S. S., An exact solution for the steady-state thermoelastic response of functionally graded orthotropic cylindrical shells. Int. J. Solids Struct., 43, 1131-1158, 2006.

[32] Arciniega, R. A., Reddy, J. N., Large deformation analysis of functionally graded shells. Int. J. Solids Struct., 44, 2036-2052, 2007.

[33] Yang, J., Shen, H. S., Free vibration and parametric resonance of shear deformable functionally graded cylindrical panels. J. Sound Vib., 261, 871-893, 2007.

[34] Jianqiao, Y., Qiujuan, M., Shan, S., Wave propagation in non-homogeneous magnetoelectro-elastic hollow cylinders. J. Ultrasonic, 48, 664-677, 2008.

[35] Abd-Alla, A. M., Nofal, T. A., Farhan, A. M., Effect of the non-homogenity on the composite infinite cylinder of isotropic material. Physics Letters A, 372, 4861-4864, 2008.

[36] Tornabene, F., Viola, E., Inman, D. J., 2-D differential quadrature solution for vibration analysis of functionally graded conical, cylindrical shell and annular plate structures. $J$. Sound Vib., 328, 259-290, 2009.

[37] Keleş, İ., Tütüncü, N., Exact analysis of axisymmetric dynamic response of functionally graded cylinders (or disks) and spheres. Journal of Applied Mechanics, 78(6), 061014 (7 pages), 2011.

[38] Shen, H. S., Xiang, Y., Nonlinear vibration of nanotube-reinforced composite cylindrical shells in thermal environments. Comput. Methods Appl. Mech. Engrg., 213, 196-205, 2012.

[39] Shen, H. S., Xiang, Y., Nonlinear vibration of nanotube-reinforced composite cylindrical panels resting on elastic foundations in thermal environments. Compos. Struct., 111, 291300, 2014.

[40] Moradi-Dastjerdi, R., Foroutan, M., Pourasghar, A., Dynamic analysis of functionally graded nanocomposite cylinders reinforced by carbon nanotube by a mesh-free method. Mater. Des., 44, 256-266, 2013.

[41] Bowman, F., Introduction to Bessel Functions, New York, Dover, 1958.

[42] Hildebrand, F. B., Advanced Calculus for Applications, Prentice-Hall. Inc. Englewood Cliffs, New Jersey, 1962.

[43] Abramowitz, M., Stegun, I. A. (Eds.), Handbook of Mathematical Functions with Formulas, Graphs and Mathematical Tables, 9th printing, New York, Dover, 1972. 